\section{Reviving Ras}

\section{By Joanne Kotz, Senior Editor}

Activated Ras proteins are among the most notorious oncoproteins, but thus far they have proven undruggable. Now, teams at Roche's Genentech Inc. unit and at Vanderbilt University School of Medicine have independently used fragment-based drug discovery to identify a new small molecule binding site that blocks Ras activation. ${ }^{1,2}$

The results could reinvigorate drug discovery efforts against the target. Remaining hurdles include developing more potent inhibitors and testing whether optimized molecules block mutant Ras-driven cancers.

Ras proteins are central regulators of cell growth and proliferation. In response to growth factors, these GTP binding proteins are turned on by guanine nucleotide exchange factors (GEFs) such as son of sevenless homolog 1 (SOS1).

Collectively, mutations in the three isoforms of Ras-HRAS ( $v$-Ha-ras Harvey rat sarcoma viral oncogene homolog), K-Ras and NRAS (neuroblastoma Ras viral ( $v$-Ras) oncogene)-occur in over $20 \%$ of all cancers.

Indeed, Guowei Fang, a senior scientist in research oncology at Genentech, noted that Ras "is the most frequently mutated oncogene in human
"This is an incredibly important advance. You can now take another look at Ras applying modern techniques, including fragments and crystallography, and hone in on trying to find inhibitors."

-John Lyons, Astex Pharmaceuticals Inc. chronic lymphocytic leukemia (CLL) and in a Phase I/II trial as a single agent in relapsed or refractory CLL or other lymphoid malignancies.

To tackle K-Ras, both the Vanderbilt and Genentech teams turned to fragment-based drug discovery.

Using this approach, small libraries of fragments that weigh 100-300 Da are screened by sensitive structural or biophysical methods that identify molecules that bind a target with high micromolar to low millimolar affinities.

In contrast, high throughput screening typically involves sifting through very large libraries of small molecules weighing 300-600 Da to identify hits with affinities or activities in the nanomolar to low micromolar range.

Fesik's team screened 11,000 fragments using NMR and identified 140 that bound to K-Ras. The Genentech team also used NMR, screening 3,300 fragments and identifying 25 confirmed hits.

In both cases, the groups obtained co-crystal structures that revealed the fragments bound at the interface with SOS1. In vitro assays showed the fragments blocked SOS1-mediated activation of K-Ras with mid- to high-micromolar IC $_{50}$ values. This newly identified binding site is conserved among isoforms, and both groups confirmed that the fragments bound to wild-type $\mathrm{K}-\mathrm{Ras}$, mutant K-Ras and HRAS.

The Genentech group's lead fragment reduced epidermal growth factor (EGF)-mediated activation of Ras proteins, which is known to be mediated by SOS1, with a mid-micromolar $\mathrm{EC}_{50}$ in human cells.

Fang added that in parallel with fragment-based screening, Genentech conducted a traditional high throughput screen of the company's full libraryover one million compounds-in a biochemical tumors and correlates with poor prognosis of patients. But from the druggability side, this target has been worked on by nearly every pharma over the last 20 years with little benefit."

Fang said advances in using fragments-pieces of small moleculesin drug discovery "made us give this a second thought."

\section{Calling on fragments}

Of the three Ras isoforms, K-Ras is the most frequently dysregulated, with activating mutations occurring in about $80 \%$ of pancreatic cancers, $40 \%$ of colon cancers and $25 \%$ of lung cancers.

Stephen Fesik, who headed the Vanderbilt team, said when he joined the university in 2009 and set out to work on six validated but challenging cancer targets, K-Ras was on the list. Fesik is professor of biochemistry, pharmacology and chemistry and chair in cancer research in the Vanderbilt University School of Medicine.

Before Vanderbilt, Fesik was divisional VP of cancer research at Abbott Laboratories. While at Abbott, Fesik led the effort to develop inhibitors of the B cell lymphoma 2 (BCL-2; BCL2) family of proteins, which are challenging protein-protein interaction targets. The resulting inhibitor, navitoclax (ABT-263), is partnered with Genentech. The compound is in a Phase II trial in combination with Rituxan rituximab in assay of Ras function. Fang said the screen yielded no workable hits.

Results from Genentech were published in the Proceedings of the National Academy of Sciences. The Vanderbilt team published its findings in Angewandte Chemie International Edition.

"These compounds are very early, but it's really very exciting that groups have identified novel fragments binding to one of the so-called undruggable targets and importantly solved crystal structures with ligands bound," said Martin Drysdale, professor and head of the drug discovery program at The Beatson Institute for Cancer Research.

He said his group also is targeting K-Ras via fragment-based approaches. The work has not yet been published. Drysdale previously was deputy research director at the fragment-based and structure-based drug discovery company Vernalis plc.

"This is an incredibly important advance. You can now take another look at Ras applying modern techniques, including fragments and crystallography, and hone in on trying to find inhibitors," said John Lyons, VP of translational research at Astex Pharmaceuticals Inc. "Pharmas will doubtless want to give this a go. It's such a high-value target that no one will want to be left out."

Lyons declined to disclose whether Astex would now be pursuing Ras proteins. 


\section{Next up, mutant K-Ras}

An open question is whether blocking Ras activation-rather than blocking Ras activity-will have an impact in mutant $K$-Ras-driven cancers.

"These results have shown that you can inhibit the activation of K-Ras but have not yet shown that you can inhibit mutant K-Ras

\section{"These compounds are very early, but it's really very exciting that groups have identified novel fragments binding to one of the so-called undruggable targets and importantly solved crystal structures with ligands bound." \\ -Martin Drysdale, \\ The Beatson Institute for Cancer Research} signaling," noted Lyons.

Although the fragments described in the papers block Ras activation, future optimized molecules could block both activation and downstream signaling.

"Where these compounds bind Ras is close to the Ras-SOS1 interface but also close to the Ras-effector interface," said Weiru Wang, a senior scientist in Genentech's structural biology department. Thus, he said it may be possible to design a molecule that also antagonizes the interaction of Ras with its various downstream effectors.

Even if an inhibitor of mutant K-Ras activity cannot be developed, a molecule that only blocks wild-type Ras may still have activity in mutant $K$-Ras-driven cancers.

For example, in mouse models of mutant $K$-Ras-driven pancreatic tumors, both mutant K-Ras and wild-type HRAS are required for tumor growth. ${ }^{3}$ Fang added that Genentech also has internal data supporting a co-dependency between mutant K-Ras and wild-type Ras in a subset of mutant $\mathrm{K}$-Ras-driven cancers. In this case, he added, a pan-selective Ras inhibitor would be the goal.

Finally, inhibitors of Ras activation could be useful in cancers with alterations upstream of Ras. Fang noted that in EGF receptor (EGFR)-driven cancers, inhibiting Ras activation would block a major downstream signaling pathway.

The Vanderbilt and Genentech teams are now working to optimizing the potency of their inhibitors and to develop inhibitors of mutant K-Ras.

Fesik said he has had discussions with companies, but "most would like to see very potent molecules that are active in cells to take down the risk. Frankly, the jury's still out on whether we can do this."

Fesik declined to disclose the patent status of the work but said the project is available for licensing or partnering.

Genentech declined to disclose the patenting status of the work. It is not available for licensing.

Kotz, J. SciBX 5(21); doi:10.1038/scibx.2012.536

Published online May 24, 2012

\section{REFERENCES}

1. Sun, Q. et al. Angew. Chem. Int. Ed.; published online May 8, 2012; doi:10.1002/anie.201201358

Contact: Stephen W. Fesik, Vanderbilt University School of Medicine, Nashville, Tenn.

e-mail: stephen.fesik@vanderbilt.edu

2. Maurer, T. et al. Proc. Natl. Acad. Sci. USA; published online March 19, 2012; doi:10.1073/pnas.1116510109

Contact: Guowei Fang, Genentech Inc., South San Francisco, Calif.

e-mail: fang.guowei@gene.com

Contact: Weiru Wang, same affiliation as above e-mail: wang.weiru@gene.com

3. Lim, K.-H. et al. Nature 452, 646-649 (2008)

\section{COMPANIES AND INSTITUTIONS MENTIONED}

Abbott Laboratories (NYSE:ABT), Abbott Park, III.

Astex Pharmaceuticals Inc. (NASDAQ:ASTX), Dublin, Calif. The Beatson Institute for Cancer Research, Glasgow, U.K. Genentech Inc., South San Francisco, Calif.

Roche (SIX:ROG; OTCQX:RHHBY), Basel, Switzerland Vanderbilt University School of Medicine, Nashville, Tenn. Vernalis plc (LSE:VER), Winnersh, U.K. 